\title{
DISTRIBUIÇÃO E INFECÇÃO DE TRIATOMÍNEOS POR TRYPANOSOMA DO TIPO CRUZI NA ILHA DE SÃO LUÍS-MARANHÃO
}

\author{
Reginaldo Peçanha Brazil, Antonio Rafael da Silva, Arnaldo Albarelli e \\ Jerônimo F. Vale
}

\begin{abstract}
São apresentados dados referentes às espécies e infecção natural de triatomíneos na Ilha de São Luis-MA. Dos triatomineos coletados foram encontradas as seguintes espécies: Rhodnius pictipes Rhodnius neglectus, Rhodnius nasutus, Triatoma rubrofasciata, Panstrongylus lignarus $e$ Panstrogylus geniculatus. A presença de infecção natural por Trypanosoma do tipo cruzi foi detectada em 19,7\% do total de triatomineos sendo o R. pictipes a espécie mais homogênea em distribuição na Ilha e com índice de infeç̧ão natural de $38,8 \%$.

Palavras chaves: Trypanosoma cruzi. Doença de Chagas. Triatomineos. Maranhão.
\end{abstract}

A doença de Chagas não é endêmica no Estado do Maranhão. Entrentanto, com a descoberta de quatro casos autóctones na Ilha de São Luis 8 nossa atenção ficou voltada para as invasões ocasionais de domicilios por triatomineos silvestres 1 .

No presente trabalho procuramos determinar as espécies de triatomineos existentes na Ilha de São Luís, bem como os indices de infecção por Trypanosoma do tipo cruzi destes hemípteros.

\section{MATERIAL E MÉTODOS}

\section{Descrição da Ilha}

A Ilha de São Luís tem uma área de $952 \mathrm{~km}^{2} \mathrm{e}$ está dividida em três municípios: São Luís, São José de Ribamar e Paço do Lumiar. E formada por terrenos baixos com vegetação litorânea intensa e crescimento de palmeiras sobressaindo principalmente o babaçu (Orbignya martiana).

Quanto ao aspecto climático é característico o tipo clima tropical úmido com média anual de temperatura em torno de $26^{\circ} \mathrm{C}$ e com variaçoes minimas de mês para mês. Ocorrem na Ilha duas estaçōes climáticas bem delimitadas: o inverno ou das chuvas, entre ianeiro e junho e o verão, de julho a dezembro. $O$ indice de pluviosidade anual situa-se acima de $2000 \mathrm{~mm}^{4}$.

\section{Captura de Triatomíneos}

Grande parte do material foi obtido por capturas ocasionais em algumas localidades e bairros adjacentes à São Luis. Parte do material foi coletado através da SUCAM sendo que poucas foram as capturas por levantamentos domiciliares.

Trabalho realizado no Departamento de Patologia da Universidade Federal do Maranhão em colaboração com a Superintendência das Campanhas de Saúde Pública (SUCAM).

Endereço para correspondência: Departamento de Parasitologia-ICB-CCS-UFRJ - Cidade Universitária - 21.941 - RJ, Brasil.

Recebido para publicação em $16 / 4 / 84$
Nos povoados de Rio Grande e Caúra, onde ocorreram os casos autóctones de doença de Chagas, foram feitos levantamentos domiciliares de triatomíneos nas 60 habitações existentes.

No laboratório procedeu-se à determinação específica dos hemipteros capturados e fez-se a pesquisa de flagelados por meio da microscopia direta das fezes. Das fezes positivas foram feitos esfregaços e posterior coloração pelo Giemsa. Em alguns casos foram feitas inoculações intraperitoneais com fezes positivas em camundongos albinos.

\section{RESULTADOS}

A procedência dos triatomíneos capturados inclui os três municipios da Ilha de São Luis cuja distribuição foi a seguinte (Fig. 1):

Rhodnius pictipes - Municipio de São Luís: Centro (10 1), Bacanga (6० 80), Jordoa (1o), Tirirical (10 2o), Anil (2o 1o), Cohab (1o), Filipinho (1o), Fátima (2. 2o), Renascença (11.3o), Sacavem (3० In), Turu (9. 13o), Olho D’Água (5o) Bequimão (19 10); Município de Paço do Lumiar: Araçagi (1\%); Municipio de Ribamar: Moropoia (1\%).

$R$. neglectus - Município de São Luís: Turu (3․ 1o), Vinhais (1. 10).

$R$. nasutus - Município de São Luís: Sacavem (1o), Cutim (1o), Coroadinho (1o), Turu (1), Alemanha (1).

$R$. robustus - Municipio de São Luís: Turu (1o), Vinhais (10).

Triatoma rubrofasciata-Municipio de São Luís: Centro (8. 1o 1n), Lira (10\% 17o 14nn), Coreia (5\% 8o $1 \operatorname{lnn}$ ), Camboa (1\%), Anil (10), Madre Deus (3\%), Vila Bessa (1\% 10), Monte Castelo (1\%), Fabril (1\%) Conjunto Fesma (10), Fátima (2\%), Vila Passos (2\%), Filipinho (2\% 1o), Bacanga (2\% 3o).

Pantrongylus lignarus - Municipio de, São Luís: Sacavem (2\%), Turu (1\% lo ln), Olho D'Água (1\%), Ipase (1\%), Fátima (1\%): Município de Paço do Lumiar: Araçagi (2\%).

Panstrongylus geniculatus - Municipio de São Luís: Turu (1\%), Vila Embratel (1\%). 
Brazil RP, Silva AR, Albarelli A, Vale FJ. Distribuiçäo e infecção de triatomineos por Trypanosoma do tipo cruzi na Ilha de São Luís-Maranhão. Revista da Sociedade Brasileira de Medicina Tropical 18: 257-260, Out-Dez, 1985

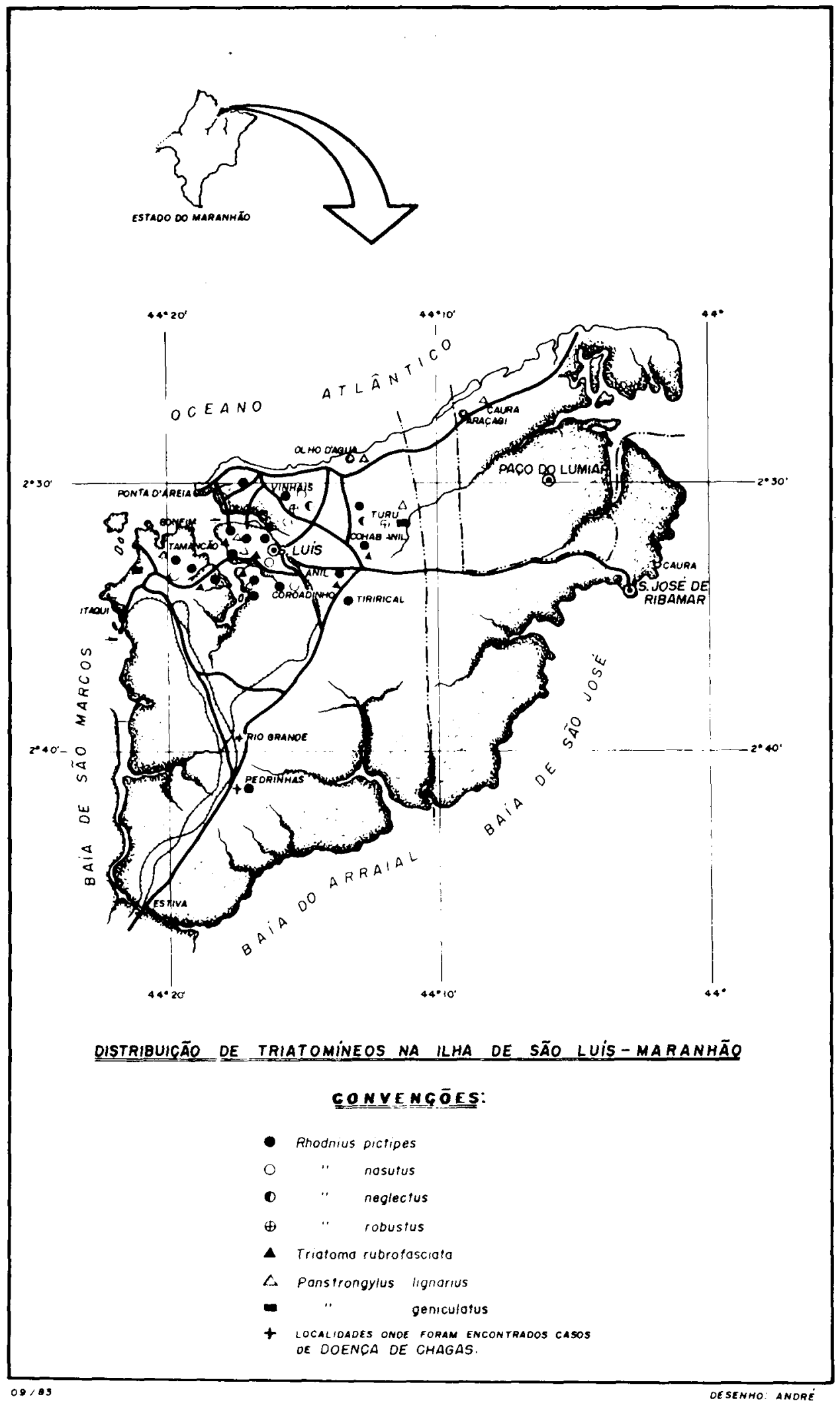


Brazil RP, Silva AR, Albarelli A, Vale FJ. Distribuição e infecção de triatomíneos por Trypanosoma do tipo cruzi na Ilha de São Luís-Maranhão. Revista da Sociedade Brasileira de Medicina Tropical 18: 257-260, Out-Dez, 1985

O Triatoma rubrofasciata foi a especie predominante com $42,7 \%$ do total dos triatomíneos examinados. A predominância da espécie está principalmente em função de sua domiciliação nos bairros centrais da cidade onde ocorrem maior número de capturas. O Rhodnius pictipes foi a única espécie com distribuição homogênea na Ilha $(41,2 \%)$, predominando principalmente nas áreas de loteamento recente como Turu, Vinhais, Renascença (Fig. 1). Além das duas espécies que predominaram no material coletado (T. rubrofasciata e $R$. pictipes) fica assinalado a ocorrência de $R$. neglectus e $R$. robustus ainda nào registrados no Estado do Maranhão.

A presença de Trypanosoma do tipo cruzi foi detectada em $19,7 \%$ dos triatomineos examinados. $O$ indice de infecção natural está assinalado na Tabela 1.

Os resultados da inoculaçăo experimental em camundongos com amostras provenientes de $R$. pictipes demonstraram uma parasitemia sempre muito baixa dificilmente visualizada pelo exame de sangue. Entretanto, o exame histopatológico revelou lesões cardiacas após 40 dias de infecção.

A maioria dos $T$. rubrosfaciata apresentava-se parasitada pelo Trypanosoma conorhini, parasito de ratos, de cujo sangue o $T$. rubrofasciata se alimenta. Entretanto, chamamos a atenção para o encontro de dois espécimes naturalmente infectados por Trypanosoma do tipo cruzi em uma pensão do Cantro de São Luís.

Tabela 1-Distribuiçāo de infecçāo natural por Trypanosoma do tipo cruzi da Ilha de São LuisMaranhão.

\begin{tabular}{lcc}
\hline Espécie & $\begin{array}{c}n . \text { infectados } \\
n . \text { examinados }\end{array}$ & $\begin{array}{c}\% \text { de } \\
\text { infecçáo }\end{array}$ \\
\hline Rhodnius pictipes & $35 / 90$ & 38,8 \\
R. neglectus & $3 / 6$ & 50,0 \\
R. nasutus & $0 / 5$ & 00 \\
R. robustus & $1 / 2$ & 50,0 \\
Triatoma rubrofasciata & $2 / 103$ & 1,9 \\
Panstrongylus lignarus & $2 / 10$ & 22,0 \\
P. geniculatus & $0 / 2$ & 00 \\
\hline Total & $43 / 218$ & 19,7 \\
\hline
\end{tabular}

\section{DISCUSSĀO}

Durante o aparecimento de 3 casos autóctones da doença de Chagas na Ilha ${ }^{2}$ os levantamentos domiciliares nas localidades de Caúra e Rio Grande nunca lograram êxito na captura de triatomíneos. Desta forma o mecanismo de transmissão na área permanece especulativo; se por via oral como sugerido por Shaw e cols ${ }^{7}$ nos casos autóctones de Belém, ou através de infecção acidental por triatomineos silvestres. A última hipótese é reforçada pela invasão ocasional de triatomineos silvestres naturamente infectados por Trypanosoma do tipo cruzi nos domicílios.
A fauna de triatomineos no Estado do Maranhào tem sido pouco estudada, já tendo sido, entretanto, encontrado, Panstrongylus megistus, $P$. geniculatus, $P$. lignarus, Triatoma sordida, T. infestans, $T$. brasiliensis, Triatoma rubrofasciata, $T$. pseudomaculata, Rhodnius brethesis, $R$. pictipes, $R$. nasutus e $R$. prolixus 2356 . No que diz respeito à Ilha de São Luis são inexistentes os dados sobre as espécies de triatomineos na regiāo. Desta forma os dados obtidos no presente trabalho ampliam o conhecimento da distribuição geográfica de várias espécies de triatomineos encontrados na região Sul e Sudeste do Estado bem como assinalam a presença de Rhodnius neglectus e $R$. robustus no Estado do Maranhão.

Chamamos a atenção para o encontro de triatomineos silvestres com alto indice de infecção natural por Trypanosoma do tipo cruz $i$ que poderão se domiciliar ampliando assim a distribuição geográfica da doença de Chagas.

\section{SUMMARY}

Data is presented on the species and infection rate of reduviid bugs collected in São Luis island, Maranhão State. The following species have been collected: Rhodnius pictipes, R. neglectus, R. nasutus, R. robustus, Triatoma rubrofasciata, Panstrongylus lignarus and $\mathrm{P}$. geniculatus. The infection rate with $\mathrm{T}$. cruzi-like trypanosomes was $19.7 \%$ of the total bugs collected. R. pictipes shows homogeneous distribution in the island and an infection rate of $38.8 \%$.

Key words: Chagas' disease. Trypanosoma cruzi. Triatominae. Maranhão.

\section{AGRADECIMENTOS}

Gostariamos de agradecer aos técnicos do Setor de Entomologia da SUCAM-MA, que muito nos ajudaram no presente trabalho e ao CNPq pelo auxílio financeiro.

\section{REFERÊNCIAS BIBLIOGRÁFICAS}

1. Brazil RP, Silva AR. Triatomine vectores of Trypanosoma cruzi-like. Trypanosoma in urban areas of São Luis, Maranhāo, Brazil. Transactions of the Royal Society of Tropical Medicine and Hygiene 77:568, 1983.

2. Castro FJ, Silveira AC. Distribuição da doença de Chagas no Brasil. Revista Brasileira de Malariologia e Doenças Tropicais 21:85-98, 1979

3. Forattini OP, Barata JMS, Santos JLF. Silveira AC. Hábitos alimentares, infecção natural e distribuição de triatomíneos domiciliados na região central do Brasil. Revista de Saúde Pública de São Paulo 16: 171-204, 1982.

4. Hueck K. As florestas da América do Sul, São Paulo, Editora Universidade de Brasilia/Editora Poligono, 1972.

5. Lucena DT. Ecologia dos triatomíneos do Brasil. Revista Brasileira de Malariologia e Doenças Tropicais 1 1: 577 $635,1969$. 